\title{
Preseptal and Orbital Cellulitis in Childhood: The Experience of Ankara Training and Research Hospital
}

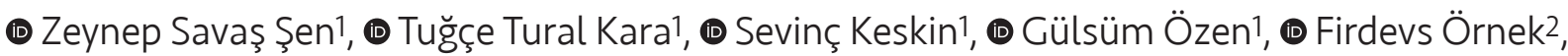 \\ (1) Bülent Alioğlu1 \\ ${ }^{1}$ Ankara Training and Research Hospital, Clinic of Paediatrics, Ankara, Turkey \\ ${ }^{2}$ Ankara Training and Research Hospital, Clinic of Ophthalmology, Ankara, Turkey
}

\begin{abstract}
Aim: Evaluation of the etiology, clinical and laboratory findings, treatment and complications of preseptal and orbital cellulitis in patients and to show that these complications can be prevented with early diagnosis and effective treatment.

Materials and Methods: Thirty-eight patients with orbital and preseptal cellulitis who had been admitted to Ankara Training and Research Hospital, Clinic of Paediatric between September 2015 and February 2017 were retrospectively studied.

Results: Thirty-five patients (92.1\%) were diagnosed with preseptal cellulitis and 3 patients (7.9\%) were diagnosed with orbital cellulitis. The mean age at diagnosis of the patients [ 24 girls (63.2\%) and 14 boys (36.8\%)] was $4.01 \pm 3.72$ years. The most frequent etiologic factor was conjunctivitis (28.9\%). Twenty-five patients $(65.7 \%)$ were treated with intravenous ampicillin-sulbactam alone as the first treatment. No patient underwent surgery. All patients recovered completely without any eye illnesses and no complications were observed.

Conclusion: Orbital infections can be healed through early diagnosis and effective antibiotic therapy in childhood and ampicillin-sulbactam therapy alone should be preferred over combination therapy due to its high effectiveness and relatively low side effects.

Keywords: Preseptal cellulitis, orbital cellulitis, child
\end{abstract}

\section{Introduction}

Orbital site cellulites are common eye infections which can be treated by the effective use of antibiotics. However, a delay in diagnosis and treatment can cause serious complications such as loss of vision, cavernous sinus thrombosis (CST), meningitis and/or sepsis (1). The major infections of the ocular adnexal and orbital tissues are defined as a preseptal cellulitis and orbital cellulitis depending on the site of the infection.

Preseptal cellulitis is characterized by swelling and redness of the orbital septum that can also spread to the upper cheek and forehead (2). On the other hand, orbital cellulitis is associated with proptosis and a limitation of eye movements. Other accompanying findings are inflammation in the conjunctiva, orbital pain, decreased visual acuity (VA), and afferent pupillary defect. Although the differences between preseptal and orbital cellulitis are understood, it is sometimes difficult to differentiate (3).

In this study, the demographic and clinical features, underlying etiology, laboratory and radiological findings, treatment and complications in patients who had been diagnosed with preseptal or orbital cellulitis during childhood were evaluated retrospectively. 


\section{Materials and Methods}

Thirty-eight patients with orbital or preseptal cellulitis who had been admitted to the Ankara Training and Research Hospital, Department of Paediatrics between September 2015 and February 2017 were retrospectively studied.

The patients with periorbital edema and/or redness, and normal eye movements were diagnosed with preseptal cellulitis, whereas at least one of following findings of limited eye movement, inflammation signs in the conjunctiva, orbital pain, decreased VA (for at least 2 lines on the Snellen chart), afferent pupillary defect signs or radiological imaging detecting inflammation in the orbital region, were accepted for a diagnosis of orbital cellulitis (1-3).

The patients' demographic, clinical features and underlying diseases such as age, gender, season of diagnosis, upper respiratory tract infection, sinusitis, dental infection, conjunctivitis, skin infection, and trauma history were obtained from the database.

The serum white blood cell count (WBC), erythrocyte sedimentation rate (ESR) and C-reactive protein (CRP) levels were recorded.

All results were evaluated using the SPSS. The collected data were analysed with respect to frequency (\%), mean \pm standard deviation, median, minimum and maximum values.

The authors obtained approval from the Ethics Committee of Ankara Training and Research Hospital with file for this study (approval number: 0002-21 on 15.03.2017). The study was conducted in accordance with the principles of the Declaration of Helsinki. The inform consent was taken from the patients' parents.

\section{Results}

Thirty-five patients (92.1\%) were diagnosed with preseptal cellulitis and 3 other patients (7.9\%) were recognized as suffering from orbital cellulitis. These diagnoses were collected from 24 female (63.2\%) and 14 male (36.8\%) individuals with a mean age of $4.01 \pm 3.72$ years old. The patients' demographic and clinical features such as age, diagnoses results, seasonal distribution, cellulitis location, hospitalization and total treatment duration are presented in Table I. By evaluation of the underlying diseases, the most common causes were listed as conjunctivitis, tooth infections, sinusitis, skin infections, upper respiratory tract infection and trauma (Table II). The patients with orbital cellulitis had decreased VA (for at least 2 lines of Snellen charts). None of the patients had preauricular lymphadenopathy.

The WBC counts were similar in preseptal (13.890 $\pm 3.485 /$ $\mathrm{mm}^{3}$, minimum: 6.900 -maximum: $21.300 / \mathrm{mm}^{3}$ ) and orbital cellulitis $\left(13.633 \pm 1.137 / \mathrm{mm}^{3}\right.$, minimum: 12.700-maximum: $14.900 / \mathrm{mm}^{3}$ ) patients, while the serum CRP and ESR levels were found to be increased in orbital cellulitis patients. The serum CRP levels were $3.91 \pm 6.18 \mathrm{mg} / \mathrm{dL}$ (minimum: 0.12-maximum: 31) in preseptal cellulitis patients and 9.64 $\pm 0.31 \mathrm{mg} / \mathrm{dL}$ (minimum: 9.4-maximum: 10) in orbital cellulitis patients. The serum ESR levels were $20.2 \pm 19.2$ $\mathrm{mm}$ /hour (minimum: 2-maximum: 78 ) in preseptal cellulitis patients and $36.3 \pm 26.4 \mathrm{~mm} /$ hour (minimum: 13-maximum: 65 ) in orbital cellulitis patients (Table III). However, statistical comparison was not applicable due to the insufficient number of patients.

Through magnetic resonance imaging (MRI) and/or computed tomography in the orbital region, 13 patients (34.2\% of all patients) were diagnosed as follows: sinusitis

Table I. Demographic and clinical characteristics of patients with orbital and preseptal cellulitis

\begin{tabular}{|c|c|c|}
\hline & Orbital cellulitis & Preseptal cellulitis \\
\hline $\begin{array}{l}\text { Average age (years) } \\
\text { (mean } \pm \text { SD) }\end{array}$ & $\begin{array}{l}10.85 \pm 2.49 \\
\text { (min: 8.19-max: 13.14) }\end{array}$ & $\begin{array}{l}3.43 \pm 3.2 \\
\text { (min: } 0.11-\max : 10.10)\end{array}$ \\
\hline Girl gender (\%) & 66.6 & 62.8 \\
\hline \multicolumn{3}{|c|}{ Cellulitis locations (\%) } \\
\hline Right & 66.6 & 40 \\
\hline Left & 33.4 & 54.3 \\
\hline Bilateral & - & 5.7 \\
\hline \multicolumn{3}{|l|}{ Eye findings (\%) } \\
\hline Edema & 100 & 100 \\
\hline Propitosis & 66.4 & 22.8 \\
\hline Discharge & - & 25.7 \\
\hline Ecchymosis & - & 14.2 \\
\hline Haemorrhage & - & 2.8 \\
\hline Redness & 33.3 & 8.7 \\
\hline Ophtalmoplegia & 66.4 & - \\
\hline \multicolumn{3}{|l|}{ Season (\%) } \\
\hline Autumn & 33.4 & 42.8 \\
\hline Winter & 66.4 & 20 \\
\hline Spring & - & 11.4 \\
\hline Summer & - & 25.7 \\
\hline $\begin{array}{l}\text { Duration of } \\
\text { hospitalization } \\
\text { (days) } \\
\text { (mean } \pm \text { SD) }\end{array}$ & $\begin{array}{l}23.33 \pm 8.62 \\
\text { (min: 14-max: } 31)\end{array}$ & $\begin{array}{l}7.71 \pm 6.93 \\
\text { (min: 3-max: 38) }\end{array}$ \\
\hline $\begin{array}{l}\text { Days of antibiotic } \\
\text { use } \\
\text { (mean } \pm \text { SD) }\end{array}$ & $\begin{array}{l}28 \pm 12.76 \\
\text { (min: } 14-\text { max: } 39 \text { ) }\end{array}$ & $\begin{array}{l}\text { 12.17 } \pm 7.15 \\
\text { (min: 4-max: 45) }\end{array}$ \\
\hline
\end{tabular}

SD: Standard deviation, min: Minimum, max: Maximum 
in 6 patients (46.1\%), subperiosteal abscess in 3 patients (23\%), preseptal cellulitis in 8 patients (61.5\%), and orbital cellulitis in 3 patients (23\%). There was evidence of sinusitis in the maxillary sinus for all the patients while 2 patients had isolated sphenoid sinusitis and another 2 children had frontal and ethmoidal sinusitis at the same time. There was one patient diagnosed with maxillary sinusitis by direct $X$-ray.

For detecting the microorganisms, blood cultures were performed on 21 patients (55.2\%) and conjunctival culture on 2 patients (5.2\%).

Of the treatment methods, 25 patients (65.7\%) were given ampicillin-sulbactam treatment $(100 \mathrm{mg} / \mathrm{kg} / \mathrm{day}$, q6hr) alone as the first option. In addition, ampicillinsulbactam treatment was given in combination with metronidazole (30 mg/kg/day, q8hr), clindamycin (30 mg/ $\mathrm{kg} /$ day, q8hr) or amikacin ( $15 \mathrm{mg} / \mathrm{kg} /$ day, q12hr) in 9 patients (23.6\%). Four patients (10.5\%) used ceftriaxone $(75 \mathrm{mg} /$ $\mathrm{kg} /$ day, q12hr) as first-line treatment. Two of these were given ceftriaxone alone and the other two were given metronidazole (30 mg/kg/day, q8hr) and vancomycin (60 $\mathrm{mg} / \mathrm{kg} /$ day, q8hr).

\section{Discussion}

If preseptal and orbital cellulitis is not properly treated, it can lead to serious complications ranging from optic neuritis, optic atrophy, blindness, CST, superior orbital fissure syndrome, orbital apex syndrome, meningitis, brain

Table II. Risk factors and associated diseases in preseptal and orbital cellulitis

\begin{tabular}{|l|l|l|}
\hline Risk factors/associated disease & Count, (n) & Percent, (\%) \\
\hline Conjunctivitis & 11 & 28.9 \\
\hline Tooth infections & 7 & 18.4 \\
\hline Sinusitis & 7 & 18.4 \\
\hline Skin infections & 5 & 13.2 \\
\hline $\begin{array}{l}\text { History of upper respiratory tract } \\
\text { infection }\end{array}$ & 5 & 13.2 \\
\hline Trauma & 4 & 10.5 \\
\hline
\end{tabular}

abscess, subdural empyema and even death. Preseptal and orbital cellulitis are more common orbital infections during childhood rather than adulthood (4-6).

Studies have shown that preseptal and orbital cellulitis have some common risk factors such as trauma and respiratory infections, cellulitis, sinusitis, tooth loss, trauma, asthma or diabetes mellitus. These findings support that low standards of hand hygiene and health play a role in the development of orbital cellulitis in developing countries (7). The most common causes of preseptal and orbital cellulitis are sinusitis, conjunctivitis, skin infections and upper respiratory tract infections (8-11).

Sinusitis is an important underlying disease for orbital infections. Sinusitis can cause orbital complications in $74-85 \%$ of cases. This condition is most commonly seen as ethmoidal sinusitis (12). In various studies, it has been shown that $85-95 \%$ of orbital infections due to sinusitis are preseptal cellulitis and 5-15\% are postseptal infections (13). In a study with 26 paediatric patients with sinusitis presenting with orbital complications, subperiosteal abscess was found in 11 patients (42.3\%), preseptal cellulitis in 13 patients (50\%) and orbital cellulitis was determined in 2 patients (7.7\%). The authors suggested that the relatively low incidence of cellulitis may be due to the fact that some of the patients are followed in children's clinics and eye clinics (14).

Similarly, the most common etiologic factor in our study was conjunctivitis. Sinusitis was detected as the second most common etiological factor. Unlike other studies in the literature, maxillary sinusitis is the most common in our patients.

The WBC count, serum CRP and ESR levels, proptosis on examination, ophthalmoplegia, decreased VA, or abnormal pupillary reflex, conjunctival discharge and/or collection or inflammation in the orbital region on radiological imaging are indicated as signs of preseptal and orbital cellulitis (8-10). In our study, the most frequent examination finding was edema. Those patients with orbital cellulitis were found to have proptosis and ophthalmoplegia, and preseptal cellulitis was the most common presentation with redness

Table III. Laboratory findings of patients

\begin{tabular}{|l|l|l|l|}
\hline & $\left(\mathbf{W B C} / \mathbf{m m}^{\mathbf{3}}\right)$ & CRP (mg/dL) & ESR (mm/hour) \\
\hline $\begin{array}{l}\text { Preseptal cellulitis } \\
\text { (mean } \pm \text { SD) }\end{array}$ & $\begin{array}{l}13890 \pm 3485 \\
\text { (min: 6900-max: 21300) }\end{array}$ & $\begin{array}{l}3.91 \pm 6.18 \\
\text { (min: 0.12-max: 31) }\end{array}$ & $\begin{array}{l}20.2 \pm 19.2 \\
\text { (min: 2-max: 78) }\end{array}$ \\
\hline $\begin{array}{l}\text { Orbital cellulitis } \\
\text { (mean } \pm \text { SD) }\end{array}$ & $\begin{array}{l}13633 \pm 1137 \\
\text { (min: 12700-max: 14900) }\end{array}$ & $\begin{array}{l}9.64 \pm 0.31 \\
\text { (min: 9.4-max: 10) }\end{array}$ & $\begin{array}{l}36.3 \pm 26.4 \\
\text { (min: 13-max: 65) }\end{array}$ \\
\hline $\begin{array}{l}\text { Total } \\
\text { (mean } \pm \text { SD) }\end{array}$ & $\begin{array}{l}13869 \pm 3352 \\
\text { (min: 6900-max: 21300) }\end{array}$ & $\begin{array}{l}4.3 \pm 6.12 \\
\text { (min: 0.12-max: 31) }\end{array}$ & $\begin{array}{l}21.5 \pm 19.92 \\
\text { (min: 2-max78) }\end{array}$ \\
\hline
\end{tabular}

WBC: White blood cell count, CRP: C-reactive protein, ESR: Erythrocyte sedimentation rate, SD: Standard deviation, min: Minimum, max: Maximum 
and discharge. The WBC count from laboratory findings was found to be similar in the preseptal and orbital cellulitis groups. CRP and ESR levels were higher in those patients with orbital cellulitis. However, there was no statistical comparison due to the insufficient number of patients. Those patients diagnosed with orbital cellulitis were screened and found to be compatible with orbital cellulitis.

It has been reported that the most frequently isolated microorganisms in blood culture and abscess cultures in patients with preseptal and orbital cellulitis are Staphylococcus Aureus and other staphylococci (7-10). In our study, no microorganisms were isolated in a total of 21 blood cultures and 2 conjunctival cultures. Furthermore, abscess drainage was not necessary in our patients; therefore, penicillinase resistant penicillin (oxacillin), ampicillinsulbactam or amoxicillin-clavunate were given as the first choice of treatment in our paediatric patients (8-11).

Streptococcus anginosus was isolated as the most frequent cause in a study with 94 paediatric patients; $34 \%$ of these patients were treated with only ampicillin-sulbactam, while the majority of patients were treated with combination therapy (cephalosporin and clindamycin or vancomycin and ampicillin-sulbactam). When increased orbital signs and/or symptoms were observed after 48 hours of treatment, the patients underwent surgery and they received vancomycin therapy. Empirical vancomycin and combination therapy are not routinely used in childhood, emphasizing the usefulness of ampicillin-sulbactam therapy in the treatment of oral cirrhosis, and also in preventing resistant organisms, drug reactions and central venous catheter infections (15).

In our study, 25 patients (65.7\%) were given ampicillinsulbactam treatment alone as the first option. In addition, ampicillin-sulbactam treatment was given in combination with metronidazole, clindamycin or amikacin in 9 patients (23.6\%). Four patients (10.5\%) used ceftriaxone as the first-line treatment. Two of these were given ceftriaxone alone and the other two were given metronidazole and vancomycin.

In our study, subperiosteal abscess developed as a complication in 3 patients. Clinical improvement was achieved with appropriate systemic antibiotic therapy without any surgical intervention. Other complications such as decreased vision and intracranial complications did not develop in any patient. Under ophthalmological examination of one patient, orbital MR imaging revealed lacrimal gland hyperplasia. The patient was evaluated for a possible tuberculosis infection and a diagnosis of tuberculosis was excluded. Ampicillin-sulbactam therapy, which had previously been used after a significant decline in eye findings after 48 hours of systemic antibiotic therapy, was replaced with intravenous piperacillin-tazobactam ( $300 \mathrm{mg} / \mathrm{kg} /$ day, 3 doses); intravenous vancomycin (40 mg/ $\mathrm{kg} / \mathrm{day}, 4$ doses) was added to the treatment when there was no adequate clinical response. Bone marrow aspiration and biopsy were performed on the patient for possible malignancy and no evidence of malignancy was found. In the third week of treatment, the patient, suffering from a high fever, underwent lumbar puncture and no pathology was detected. On the $25^{\text {th }}$ day of treatment, drug eruption was considered on the basis of widespread maculopapular rash development starting from the face and proximal to the extremity and vancomycin therapy was discontinued and intravenous teicoplanin treatment $(12 \mathrm{mg} / \mathrm{kg}$ dose 3 doses with 12 -hour intervals followed by $6 \mathrm{mg} / \mathrm{kg} /$ day single dose) was begun. After teicoplanin treatment, the fever did not reoccur and the lesion became markedly regressed. During the $4^{\text {th }}$ week of treatment, the patient whose eye symptoms had improved, was discharged from the hospital.

After discharge, $9(23.6 \%)$ patients were treated with antibiotic therapy. Two of them (22.2\%) were discharged with oral ampicillin-sulbactam, 5 patients $(55.5 \%)$ with oral amoxicillin-clavunate, and 2 patients (22.2\%) with oral ciprofloxacin and oral metronidazole combination. Acyclovir was also added to the treatment due to preseptal cellulitis, which was caused by zona zoster (Figure 1).

All patients underwent ophthalmology consultations at the ophthalmology department, and no surgical intervention was performed.

When the complications were evaluated, subperiosteal abscess was determined in 3 patients (7.89\%). In these 3 patients, the abscess was seen incidentally during orbital imaging, and no clinical deterioration was observed in the patients. Figure 2 shows an orbital MR image of a

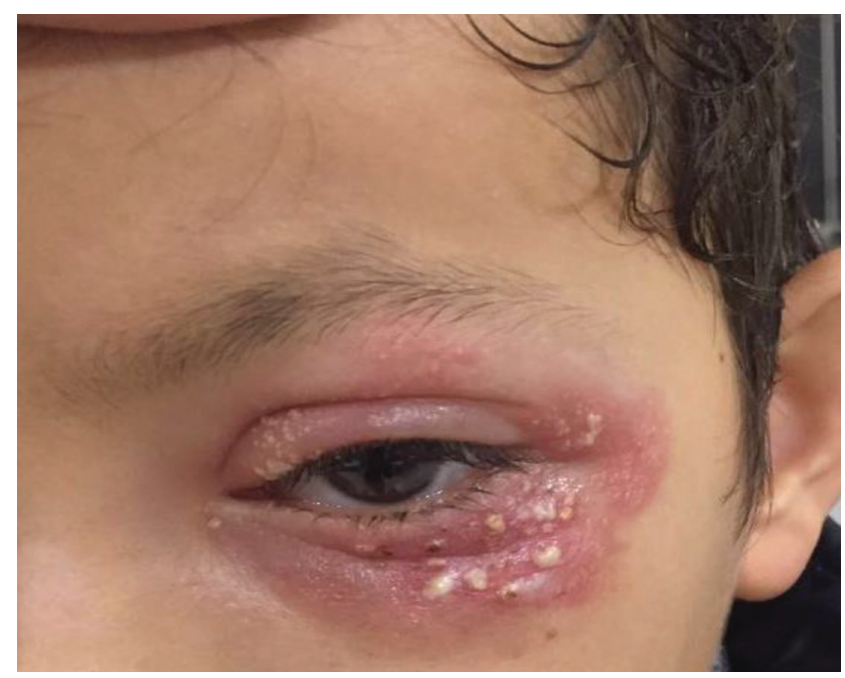

Figure 1. Ten-year-old boy with primary Herpes infection. Left periorbital erythema, edema and infected vesicular lesions 

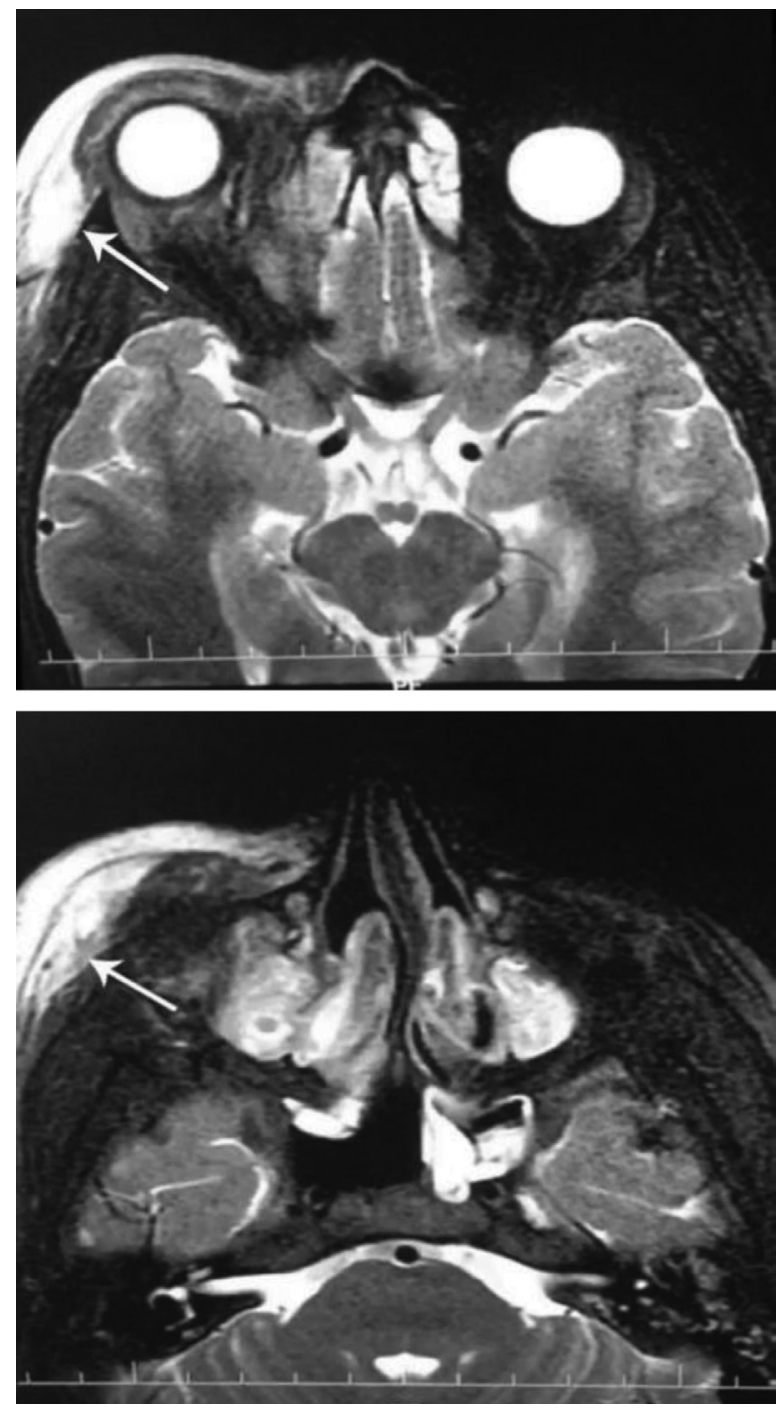

Figure 2. 11-year-old girl. Orbital magnetic resonance image of a patient with orbital cellulitis. A subperiosteal abscess of $28 \times 9 \mathrm{~mm}$ dimensions is noted at the lateral extra-coronal area, which indented the lateral rectus muscle

patient who had been admitted to our clinic with orbital cellulitis at the age of 11 and who had a subperiosteal abscess on the orbital MR. This patient, who had been given clindamycin and ceftriaxone therapy, was determined not to have to undergo surgical abscess drainage due to regression in the clinical and laboratory findings. In the other 2 patients who developed subperiosteal abscess, drug eruption occurred due to ampicillin-sulbactam treatment. Consequently, one of these patients was given intravenous clindamycin (30 mg/kg/day, 3 doses) while discontinuing ampicillin-sulbactam therapy and the other patient was given intravenous ciprofloxacin (30 mg/kg/day, 2 doses) and intravenous metronidazole (30 mg/kg/day, 3 doses) was initiated. These two patients were discharged with the resolution of the rash. In a vancomycin-treated patient, extensive erythematous lesions developed and these lesions improved when the treatment was replaced with teicoplanin. The diagnosis of subperiosteal abscess was not made with the diagnosis of deterioration in the clinical findings of the other patient, and the orbital MRI made the diagnosis based on the appearance of an abscess. After the patient was diagnosed with subperiosteal abscess, he underwent consultations at the ophthalmology and neurosurgery departments; however, no surgical drainage was performed.

\section{Conclusion}

Orbital infections in childhood can be treated with early diagnosis and effective antibiotic treatment. However, the possibility of serious complications should be kept in mind in every patient. It should be taken into account that ampicillin-sulbactam therapy alone can be used as the preferred and initial treatment option rather than combination therapy due to its high efficacy and relatively low side effects.

\section{Ethics}

Ethics Committee Approval: The authors obtained approval from the Ethics Committee of Ankara Training and Research Hospital with file for this study (approval number: 0002-21 on 15.03.2017).

Informed Consent: The inform consent was taken from the patients' parents.

Peer-review: External and internal peer-reviewed.

\section{Authorship Contributions}

Concept: Z.S.Ş., Design: Z.S.Ş., Data Collection or Processing: Z.S.Ş., T.T.K., S.K., G.Ö., F.Ö., B.A., Analysis or Interpretation: Z.S.Ş. T.T.K., B.A., F.Ö., Literature Search: Z.S.Ş., Writing: Z.S.Ş.

Conflict of Interest: No conflict of interest was declared by the authors.

Financial Disclosure: The authors declared that this study received no financial support.

\section{References}

1. Liu IT, Kao SC, Wang AG, Tsai CC, Liang CK, Hsu WM. Preseptal and orbital cellulitis: A 10-year review of hospitalized patients. I Chin Med Assoc 2006;69:415-22.

2. Jain A, Rubin PA. Orbital cellulitis in children. Int Ophthalmol Clin 2001;41:71-86.

3. Connell B, Kamal Z, McNab AA. Fulminant orbital cellulitis with complete loss of vision. Clin Exp Ophthalmol 2001;29:260-1.

4. Howe L, Jones NS. Guidelines fort he management of periorbital cellulitis/abscess. Clin Otolaryngol Allied Sci 2004;29:725-8.

5. Israele V, Nelson JD. Periorbital and orbital cellulitis. Pediatr Infect Dis | 1987;6:404-10. 
6. Jackson K, Bekar SR. Clinical implications of orbital cellulitis. Laryngoscope 1986;96:568-74.

7. Uy HS, Tuaňo PMC. Preseptal and orbital cellulitis in a developing country. Orbit 2007;26:33-7.

8. Devrim I, Kanra G, Kara A, et al. Preseptal and orbital cellulitis: 15-year experience with sulbactam ampicillin treatment. The Turkish Journal of Pediatrics 2008;50:214-8.

9. Bagheri A, Tavakoli M, Aletaha M, Salour H, Ghaderpanah M. Orbital and preseptal cellulitis: a 10-year survey of hospitalized patients in a tertiary eye hospital in Iran. Int Ophthalmol 2012;32:361-7.

10. Liu IT, Kao SC, Wang AG, Tsai CC, Liang CK, Hsu WM. Preseptal and orbital cellulitis: A 10-year review of hospitalized patients. I Chin Med Assoc 2006;69:415-22.
11. Daoudi A, Ajdakar S, Rada N, Draiss G, Hajji I, Bouskraoui M. Orbital and periorbital cellulitis in children. Epidemiological, clinical, therapeutic aspects and course. I Fr Ophtalmol 2016;39:609-14.

12. Moloney IR, Badham NJ, McRae A. The acute orbit. Preseptal (periorbital) cellulitis, subperiosteal abscess and orbital cellulitis due to sinusitis. J Laryngol Otol Suppl 1987;12:1-18.

13. Smith TF, O'Day D, Wright PF. Clinical implications of preseptal (periorbital) cellulitis in childhood. Pediatrics 1978;62:1006-9.

14. Kinis V, Ozbay M, Bakir S, et al. Management of orbital complications of sinusitis in pediatric patients. J Craniofac Surg 2013:24:1706-10.

15. Seltz B, Smith J, Durairaj VD, Enzenauer R, Todd J. Microbiology and antibiotic management of orbital cellulitis. Pediatrics 2011;127:566-72 